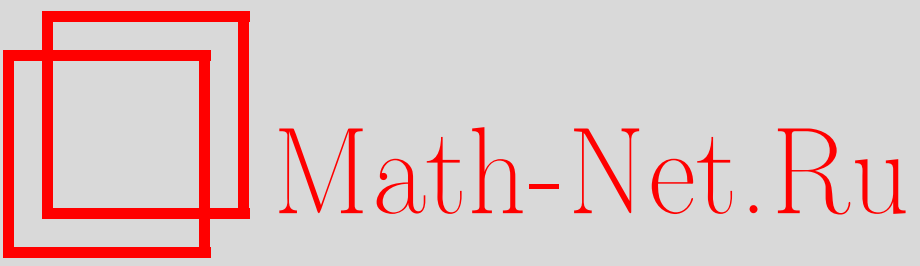

В. В. Балащенко, Канонические $f$-структуры гиперболического типа на регулярных Ф-пространствах, УМН, 1998, том 53, выпуск 4, 213-214

DOI: https://doi.org/10.4213/rm60

Использование Общероссийского математического портала Math-Net.Ru подразумевает, что вы прочитали и согласны с пользовательским соглашением

http://www.mathnet.ru/rus/agreement

Параметры загрузки:

IP: 3.89 .185 .249

26 апреля 2023 г., 16:37:26 


\title{
КАНОНИЧЕСКИЕ $f$-СТРУКТУРЫ ГИПЕРБОЛИЧЕСКОГО ТИПА НА РЕГУЛЯРНЫХ Ф-ПРОСТРАНСТВАХ
}

\author{
В. В. БАЛАЩЕНКО
}

Аффиинорные структуры на гладких многообразиях, т.е. гладкие тензорные поля типа $(1,1)$, прочно стали важнейшими объектами исследований в современной дифференциалшной геометрии. При этом толњко почти комплексным структурам (в частности, почти эрмитовым) посвящено огромное число исследований (см., например, [1]), среди которых обозначилась серия перспективных приложений. В то же время в ряде случаев возникают существенные трудности, связанные с отсутствием примеров (в особенности, инвариантных) того или иного типа структур.

Оказалось [2], что однородные $\Phi$-пространства (обобщенные симметрические пространства [3]) обладают богатым запасом инвариантных афффинорных структур, которые каноническим образом строятся по автоморфизму $\Phi$. В работе [2] для регулярных $\Phi$-пространств полностью описаны все канонические аффинорные структуры следующих классических типов: почти произведения $P$, почти комплексные $J$ и, более общо, $f$-структуры в смысле К. Яно $\left(f^{3}+f=0\right)$. В частности, для однородных $\Phi$-пространств порядка $n\left(\Phi^{n}=\mathrm{id}\right)$ получены точные вычислителшные формулы.

В данной заметке для произвольных регулярных $\Phi$-пространств охарактеризованы все канонические структуры $f$, удовлетворяющие условию $f^{3}-f=0$, т.е. включающие структуры почти произведения. Следуя [4], будем назьвать такие структуры $f$-структурами гиперболического типа. Впервые эти структуры возникли, по-видимому, в работе [5] и впоследствии изучались и обобщались рядом авторов (см., например, [6]-[8]).

Пусть $G / H$ - однородное регулярное $\Phi$-пространство [9], где $\Phi$ - автоморфизм связной группы Ли $G, \mathfrak{g}=\mathfrak{h} \oplus \mathfrak{m}-$ каноническое редуктивное разложение алгебры Ли $\mathfrak{g}$ группы $G$, соответствующее автоморфизму $\varphi=d \Phi_{e}[9]$. Пусть далее $\theta$ - ограничение $\varphi$ на инвариантном подпространстве $\mathfrak{m}, s$ - число неприводимых над $\mathbb{R}$ множителей минимального многочлена оператора $\theta$. Как обычно, отождествим $\mathfrak{m}$ с касательным пространством к $G / H$ в точке $o=H$.

Напомним [2], что инвариантная аффиннорная структура $F$ на регулярном $\Phi$-пространстве $G / H$ назьвается канонической, если представляющий ее в точке $o$ афффинор $F_{o}$ является полиномом от $\theta$. Все канонические афффинорные структуры инвариантны, кроме того, относителшно "симметрий" многообразия $G / H$, порождаемых автоморфизмом $\Phi$, и образуют коммутативную подалгебру $\mathscr{A}(\theta)$ в алгебре всех инвариантных афффинорных структур. Заметим, что в случае симметрического пространства $\left(\Phi^{2}=\mathrm{id}\right)$ подалгебра $\mathscr{A}(\theta)$ тривиальна.

Теорема 1. На любом регулярном Ф-пространстве $G / H$ имеется $3^{s}$ различных канонических $f$-структур гиперболического типа. Все такие структуры образуют (коммутативную) полугруппу в алгебре $\mathscr{A}(\theta)$, которая содержит подгруппу порядка $2^{s}$ канонических структур почти произведения.

Как известно [9], регулярные $\Phi$-пространства включают все однородные $\Phi$-пространства порядка $n$. Для таких пространств можно указать явные формулы, позволяющие вычислить все канонические $f$-структуры гиперболического типа. Условимся при этом для простоты обозначать через $f$ как саму структуру, так и ее значение в точке $o$.

Теорема 2. Пусть $G / H$ - однородное Ф-пространство порядка $n$. Все канонические $f$-структуры гиперболического типа на $G / H$ могут быть заданы полиномами $f=$ $\sum_{m=0}^{n-1} \alpha_{m} \theta^{m}$, əде:

a) если $n=2 k+1$, mo $\alpha_{m}=\alpha_{n-m}=\frac{2}{n} \sum_{j=1}^{k} \xi_{j} \cos \frac{2 \pi m j}{n}$;

б) если $n=2 k$, mо $\alpha_{m}=\alpha_{n-m}=\frac{2}{n} \sum_{j=1}^{k-1} \xi_{j} \cos \frac{2 \pi m j}{n}+(-1)^{m} \frac{\xi_{k}}{n}$.

Здесь числа $\xi_{j}, j=1,2, \ldots, k$, принимают значения из множества $\{-1 ; 0 ; 1\}$, при этом полиномь $f$ задают канонические структурь почти произведения тогда и только тогда, когда все $\xi_{j} \in\{-1 ; 1\}$. 
Детализируем ситуацию, например, для случая $n=4$ (случай $n=3$ для этих структур тривиален: $f= \pm \mathrm{id})$.

СледСтвиЕ. На однородном Ф-пространстве порядка 4 есть 3 (с точностью до знака), вообще говоря, нетривиальные канонические $f$-структуры гиперболического типа:

$$
f_{1}=P=\theta^{2}, \quad f_{2}=\frac{1}{2}\left(1-\theta^{2}\right), \quad f_{3}=\frac{1}{2}\left(1+\theta^{2}\right) .
$$

При этом операторь $f_{2}$ и $f_{3}$ являются парой взаимно дополнительных проекторов: $f_{2}+f_{3}=1, f_{2}^{2}=f_{2}, f_{3}^{2}=f_{3}$. Кроме того, следующие условия әквивалентны:

1) $-1 \notin \operatorname{spec} \theta$;

2) структура $P$ тривиальна $(P=-\mathrm{id})$;

3) структура $f_{2}$ тривиальна $\left(f_{2}=\mathrm{id}\right)$;

4) структура $f_{3}$ нулевая.

Заметим, что ряд геометрических фактов о канонических структурах $P$ для случаев $n=4,5$ получен в [11], [12].

Пусть теперь $(M, g, f)$ - гладкое многообразие с (псевдо-)римановой метрикой $g$ и $f$-структурой гиперболического типа. Пару $(g, f)$ назовем (псевдо-)римановой $f$-структурой гиперболического типа, если $g(f X, Y)=g(X, f Y)$ для всех векторных полей $X, Y$ на $M$. Отметим, что в случае $f=P$ приходим к понятию (псевдо-)римановой структуры почти произведения [13].

Если группа Ли $G$ полупроста, то на регулярном $\Phi$-пространстве $G / H$ возникает [9] инвариантная относительно группы $G$ и "симметрий" $G / H$ (псевдо-)риманова естественно редуктивная метрика $g$. Такая метрика порождается формой Киллинга алгебры Ли $\mathfrak{g}$ и именуется стандартной.

ТеОрема 3. Пусть $(G / H, g)$ - однородное Ф-пространство порядка $n$ полупростой группь Ли $G$ со стандартной метрикой $g$. Все канонические $f$-структуры гиперболического типа на $G / H$ являются относительно $g$ (псевдо-)римановыми $f$-структурами гиперболического типа.

\section{СПИСОК ЛИТЕРАТУРЫ}

[1] Gray A., Hervella L. M. // Ann. Mat. Pura Appl. (4). 1980. V. 123. № 4. P. 35-58. [2] Балащенко В. В., Степанов Н. А. // Матем. сб. 1995. Т. 186. № 11. С. 3-34. [3] Ковальский О. Обобщенные симметрические пространства. М.: Мир, 1984. [4] Кириченко В.Ф. // Итоги науки и техн. Проблемы геометрии. Т. 18. М.: ВИНИТИ, 1986. С. 25-71. [5] Okubo Т. // Ann. Mat. Pura Appl. 1966. V. 2. P. 29-44. [6] Sato I. // Tensor. 1976. V. 30. № 3. P. 219-224. [7] Adati T., Miyazawa T. // TRU Math. 1977. V. 13. № 2. P. 27-39. [8] Das L. S. // Internat. J. Math. Math. Sci. 1996. V. 19. № 1. Р. 125-130. [9] Степанов Н. А. // Изв. вузов. Сер. Матем. 1967. №3. С. 88-95. [10] Феденко А. С. Пространства с симметриями. Минск: Изд-во Белорусского ун-та, 1977. [11] Балашенко В. В., Дашевич О. В. // УМН. 1994. Т. 49. № 4. С. 153-154. [12] Балащенко В. В., Чурбанов Ю. Д. // УМН. 1990. Т. 45. № 1. С. 169-170. [13] Yano K., Kon M. Structures on Manifolds. Singapore: World Scientific, 1984. 